\title{
Plasma line observations from the EISCAT Svalbard Radar during the International Polar Year
}

\author{
Nickolay Ivchenko ${ }^{1}$, Nicola M. Schlatter ${ }^{1}$, Hanna Dahlgren ${ }^{1,2}$, Yasunobu Ogawa ${ }^{3}$, Yuka Sato ${ }^{3}$, and \\ Ingemar Häggström ${ }^{4}$ \\ ${ }^{1}$ School of Electrical Engineering, Royal Institute of Technology KTH, Stockholm, Sweden \\ ${ }^{2}$ School of Physics and Astronomy, University of Southampton, Southampton, UK \\ ${ }^{3}$ National Institute of Polar Research, Tokyo, Japan \\ ${ }^{4}$ EISCAT Scientific Association, Kiruna, Sweden
}

Correspondence to: Nickolay Ivchenko (nickolay@kth.se)

Received: 16 March 2017 - Revised: 18 September 2017 - Accepted: 19 September 2017 - Published: 24 October 2017

\begin{abstract}
Photo-electrons and secondary electrons from particle precipitation enhance the incoherent scatter plasma line to levels sufficient for detection. When detectable the plasma line gives accurate measure of the electron density and can potentially be used to constrain incoherent scatter estimates of electron temperature. We investigate the statistical occurrence of plasma line enhancements with data from the highlatitude EISCAT Svalbard Radar obtained during the International Polar Year (IPY, 2007-2008). A computationally fast method was implemented to recover the range-frequency dependence of the plasma line. Plasma line backscatter strength strongly depends on time of day, season, altitude, and geomagnetic activity, and the backscatter is detectable in $22.6 \%$ of the total measurements during the IPY. As expected, maximum detection is achieved when photo-electrons due to the Sun's EUV radiation are present. During summer daytime hours the occurrence of detectable plasma lines at altitudes below the F-region peak is up to $90 \%$. During wintertime the occurrence is a few percent. Electron density profiles recovered from the plasma line show great detail of density variations in height and time. For example, effects of inertial gravity waves on the electron density are observed.
\end{abstract}

Keywords. Electromagnetics (instrumentation and techniques)

\section{Introduction}

Incoherent scatter radars are ground-based instruments for measuring the properties of the ionospheric plasma. The radars observe the Doppler-shifted ionospheric backscatter which contains the signature of ion acoustic waves and Langmuir waves (Dougherty and Farley, 1960). The narrow part of the spectrum, typically several kilohertz, around the transmitter frequency which contains the signature of ion acoustic waves is referred to as the ion line, whose spectral shape contains information about ionospheric plasma parameters such as electron density and electron and ion temperatures. However, the estimates of plasma parameters from the ion line are often ambiguous, as they are dependent on a correct assumption of the ionospheric ion composition. The backscatter from Langmuir waves is Doppler-shifted by a few megahertz, roughly the plasma frequency, and is referred to as the plasma line. A recent review gives further details of theoretical and observational aspects of plasma line in incoherent scatter radar context (Akbari et al., 2017).

Although the plasma line is too weak to be detected when the plasma is Maxwellian, suprathermal electron fluxes can excite the Langmuir waves and consequently the plasma line to levels sufficient for detection (e.g. Nilsson et al., 1996; Guio and Lilensten, 1999). The Doppler shifts of the plasma lines from the transmitter frequency are nearly equal to the plasma frequency, so that plasma line measurements provide an accurate way to determine the electron density in the plasma volume directly from the frequency shift. Vierinen et al. (2016) used the plasma line measurements from the Sondrestrom incoherent scatter radar to determine plasma 
density profiles at very high temporal resolution in order to study the variability of the energy and flux of the electron precipitation. A coded long-pulse plasma line technique for incoherent scatter radars has been developed to obtain measurements of the plasma line at very high range and time resolution, and its strength was demonstrated in a study of small density fluctuations in the ionosphere caused by gravity waves (Djuth et al., 1994, 1997).

It has also been shown that the plasma lines can be used to determine the electron temperature in the plasma, independent of the ion line estimates (Hagfors and Lehtinen, 1981; Kirkwood and Bjorna, 1992). Nicolls et al. (2006) used the found asymmetry of up- and downshifted plasma lines to accurately determine the electron temperature profile above Arecibo at high resolution. The same asymmetry can also provide information on ionospheric currents, since the Doppler shift of the electrons depend on the electron drift (e.g. Bauer et al., 1976; Behnke and Ganguly, 1986).

In this paper we present an algorithm for extracting plasma line frequency profiles from recorded incoherent scatter radar observations. We apply the method to data from the European Incoherent Scatter Scientific Association (EISCAT) Svalbard Radar (ESR) during the International Polar Year (IPY, 20072008) and investigate the statistical occurrence of plasma line enhancements.

\section{Method}

An alternating code experiment (Lehtinen and Häggström, 1987) was run almost continuously at ESR during the IPY. The experiment uses an alternating code with 30 bits, each $30 \mu$ s long, and a code cycle of 64 pulses with a code cycle length of $240 \mathrm{~ms}$ and an inter-pulse period of $3.75 \mathrm{~ms}$. In addition to the ion line data, the experiment contains two receiver bands offset from the transmitter band, covering \pm (3.1 to 4.8$) \mathrm{MHz}$ at $1.1 \mathrm{kHz}$ resolution, to observe up- and downshifted plasma lines. These plasma line channels are sampled at $0.6 \mu$ s and data are stored as lag profiles which are integrated over $6 \mathrm{~s}$. The measurements cover a range of 127 to $278 \mathrm{~km}$, with a range resolution of $4.5 \mathrm{~km}$.

During the IPY about 3.5 million data files, each corresponding to a $6 \mathrm{~s}$ interval, were recorded for the two plasma line channels. In order to process this large data set the algorithm is required to have a short runtime for each processed spectrum such that the entire dataset can be processed within a few days.

Alternating codes are used to achieve high range resolution of the measurements while using a high transmission duty cycle for high radar efficiency. The alternating code experiments are designed for targets which can be assumed to be stationary over the transmission of a code cycle. For stationary backscatter targets the range-Doppler ambiguity function (Van Trees, 2004) is a sharp peak well defined by the code. For time variable targets, however, the spread of the ambiguity function increases and signal power is effectively lost to points in the range-Doppler space other than the target. Langmuir waves in the ionosphere, and especially in the auroral ionosphere, are such targets with high temporal variability. Therefore, the radar plasma line when observed with alternating code experiments often exhibits unwanted signal contributions referred to as clutter.

In order to effectively recover the shape of the plasma line, an algorithm needs to be designed to remove this clutter. Since the time variability of the plasma line backscatter is an unknown property the effective range-Doppler ambiguity function is not known. Therefore deconvolution or more general methods assuming a fixed global ambiguity function are not suitable to recover the plasma line range-frequency dependence from the measurements.

The method outlined here is based on thresholding of the plasma line spectrum to detect points in range frequency for which the plasma line is enhanced. The effect of clutter is reduced by removing the median power for each range gate and frequency bin as outlined below. For each range gate a weighted mean frequency is calculated over selected frequency bins. In the following the steps of the method are outlined and described by the help of a data example recorded on 17 June 2007 at 07:00:06 UT, and shown in Fig. 1:

\section{A. Computation of the plasma line spectra}

The auto-correlation function measured with the radar for each range gate is stored for both the up- and downshifted plasma line channels. We use the first 80 complex-valued lags of the autocorrelation function (at $0.6 \mu$ s sampling), with a Hamming window, to produce plasma line spectra. The resulting frequency resolution of the spectra is $10 \mathrm{kHz}$.

In step A time integration can be applied by integrating the auto-correlation function over consecutive measurements. For the IPY data the resulting time resolution is multiples of $6 \mathrm{~s}$.

B. Median filtering of the spectra

To reduce noise a median filter is applied to the upand downshifted spectra with a frequency width of nine points corresponding to $10 \mathrm{kHz}$ width. The resulting upshifted plasma line spectrum is shown in Fig. 1 (B).

C. Removal of noise and clutter

Background variations in the noise level are removed in two steps:

C1. For each frequency bin the median is calculated along the range gates and subtracted from each of the contributing points. The resulting up-shifted plasma line spectrum is shown in Fig. 1 (C1).

$\mathrm{C} 2$. For each range gate the median of the spectrum is subtracted. The resulting up-shifted plasma line spectrum is shown in Fig. 1 (C2). 

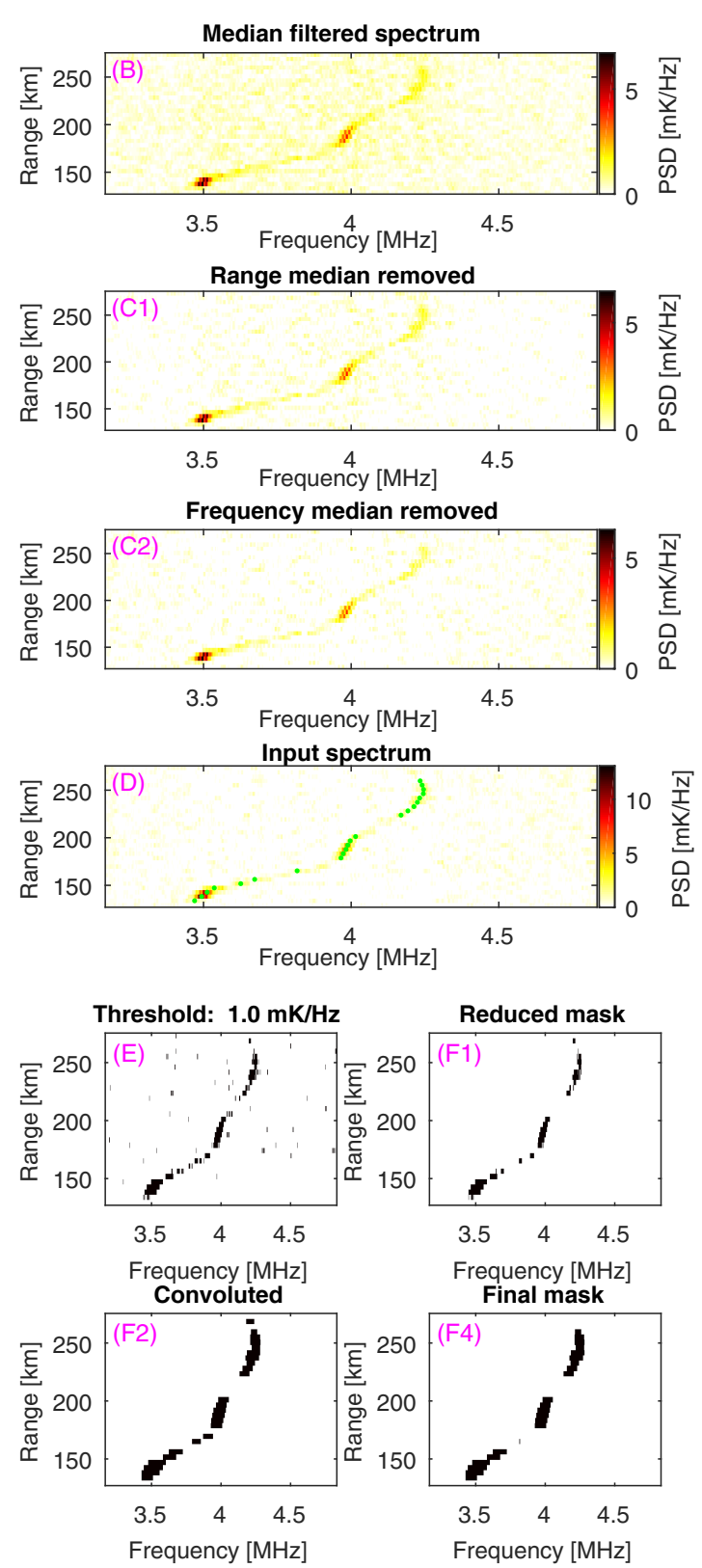

Figure 1. Example of reduction of plasma line data from 17 June 2007 at 07:00:06 UT.

D. Averaging up- and downshifted plasma line spectra

In the IPY experiment the plasma line is recorded for up- and downshifted frequencies. Since any frequency shifts of the plasma lines due to their different wave numbers and strong currents would be less than or up to the spectral resolution of the IPY measurements, an average plasma line spectrum can be computed from the up- and downshifted spectra to increase the signal-tonoise ratio (SNR). In Fig. 1 (D) the resulting average spectrum is shown.
E. Thresholding of the spectrum

Points with power above the background level trace out an altitude profile of the plasma frequency. A suitable threshold must be selected to recover large parts of the profile, while rejecting the outliers due to noise. The approach taken here is to use a low detection threshold in combination with subsequent elimination of falsely selected noise points. Thresholding is done in terms of the median absolute deviation, MAD. The MAD is more robust to outliers, which the features to be extracted represent, than the standard deviation. It is defined as

$\mathrm{MAD}=\operatorname{median}_{r, f}\left(\left|S_{r, f}-\operatorname{median}_{r, f}\left(S_{r, f}\right)\right|\right)$.

Here $S_{r, f}$ is the spectral density for a given frequency $f$ and range $r$ and the two medians are calculated over all points. To introduce a threshold that is robust against noise, but low enough to not reject too many points, a factor of 4.2 has been found to be useful, i.e. the threshold is

threshold $=4.2 \cdot$ MAD.

For normally distributed data this threshold corresponds to $3 \sigma$. In the data example discussed here threshold $=1.0 \mathrm{mK} \mathrm{Hz}^{-1}$. Figure 1 (E) shows the binary mask, which is defined by points of $S_{r, f}$ which exceed the median of $S_{r, f}$ by the threshold. As one can see the binary mask still includes points due to noise, which are removed in the following steps.

F. Further reduction of falsely selected points

To reduce the number of falsely selected points the previously calculated mask is reduced by the following steps:

F1. Removal of small areas

The mask is reduced by areas with less than 15 contributing points.

F2. Convolution with kernel

The mask is then convoluted by a structuring kernel, with the size of one range gate and 20 frequency bins, to fill the gap between nearby areas.

F3. Removal of small areas

The mask is reduced by areas with less than 140 contributing points.

F4. Final mask

To the final mask points which exceed the median by $1.8 \cdot$ threshold are added. This step guarantees that strong features, which might otherwise be removed in step F1 and F3, are included in the final mask. This step has proven to be useful especially for features where the plasma frequency has a sharp local maximum or minimum, such as the often occurring local minimum in plasma frequency just below $150 \mathrm{~km}$ range. 
The effect of steps F1-F4 on the mask is shown in the bottom four panels of Fig. 1.

G. Computation of the plasma line frequency

For each range gate the plasma frequency is computed by using the weighted average of the plasma line spectrum over frequency bins selected by the final mask. For this step the spectrum computed in step D, i.e. the average spectrum of the up- and downshifted plasma line channel, is used. In Fig. 1 (D) the estimated plasma line profile is shown by green points.

When the plasma line backscatter is strong and the plasma line frequency is outside the sampled frequency bandwidth aliasing might occur. That means that plasma line backscatter at, for example, $3 \mathrm{MHz}$ is observed at 4.6 MHz. The implemented algorithm attempts to correct for aliasing by analysing the range-frequency dependence of the plasma line (not outlined here).

\section{H. Computation of the plasma density}

In the last step the plasma density is computed from the plasma line frequency. The frequency of Langmuir waves depends on the electron density and the electron temperature. The linear dispersion relation for Langmuir waves in the direction of the magnetic field is $\omega^{2}=\omega_{p}^{2}+k^{2} v_{\mathrm{th}}^{2}$, where $v_{\mathrm{th}}$ is the electron thermal velocity and $\omega_{p}$ the plasma frequency. The electron thermal velocity is calculated from the electron temperature found by standard ion line analysis.

\section{Results}

The developed algorithm was run on the IPY dataset, from 21 May 2007 to 6 February 2008, using a 30 s integration of plasma line data. Plasma line backscatter is detectable in $22.6 \%$ of the range gates. However, the detectability has strong dependence on season, time of day, and range. Figure 2 shows the percentage of detected plasma line backscatter as a function of altitude and time of day for three time periods. During May-July, panel (a), the occurrence is highest reaching up to $90 \%$ between altitudes of 150 and $200 \mathrm{~km}$ prenoon. The F-region peak, at altitudes near $220 \mathrm{~km}$ and above, has lower occurrence, reaching a maximum of $80 \%$. During August-October, panel (b), the occurrence decreases and highest occurrence is achieved for the F-region peak around 09:00 UT with 70\%. During November-February, panel (c), when there is no or little photo-ionization the occurrence drops significantly and the plasma line is detected more sporadically. Detections during this period occur mainly between 02:00 and 07:00 and between 12:00 and 16:00 UT with an occurrence rate of around $10 \%$. Around local magnetic noon, 09:30 UT, a population of plasma line detection around $240 \mathrm{~km}$ altitude is observed. As ESR is located at high geomagnetic latitude, and the ionosphere is in the dark during
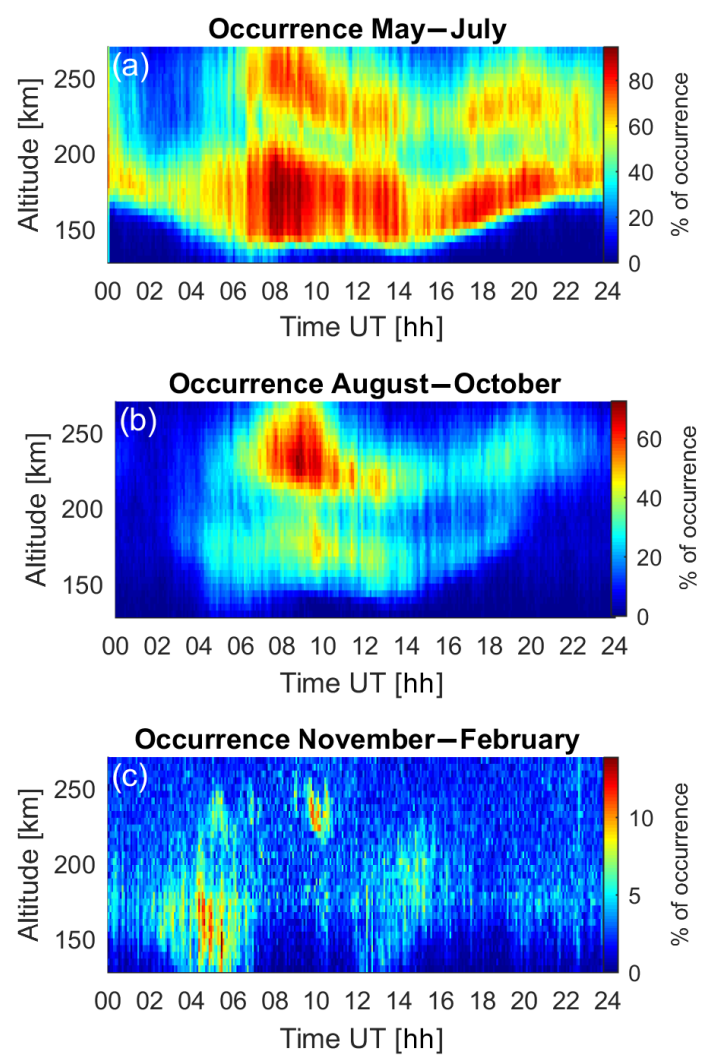

Figure 2. Occurrence of detected plasma line backscatter with $30 \mathrm{~s}$ integration: (a) May to July 2007; (b) August to October 2007; (c) November 2007 to February 2008. Magnetic local time at ESR is $\mathrm{UT}+3.1 \mathrm{~h}$.

the winter season, neither conjugate photoelectrons nor local production can account for the enhancement of the plasma line, which has to be related to particle precipitation.

Plasma density profiles computed from plasma line analysis neglecting electron pressure are shown in Fig. 3 for 17 June 2007. In panel (a) data for the whole day are shown. From about 06:00 to 12:00 UT the data coverage is good due to the presence of photoelectrons in the ionosphere. Between 00:00 and 06:00 UT as well as between 12:00 and 24:00 UT the coverage is sporadic. During these intervals particle precipitation occurs and the electron temperature above $200 \mathrm{~km}$ altitude varies between 2000 and $3000 \mathrm{~K}$. The sporadic plasma line detection during these intervals might be due to either photo-electrons or secondary electrons from particle precipitation; however, we do not attempt to investigate the mechanisms here. In Fig. $3 b$ a shorter time period of the same data is shown. The electron density recovered from the plasma line shows great detail in time, but also altitude. Structures propagating towards the radar, i.e. decreasing in altitude, are clearly visible. These structures in electron density are the effect of travelling ionospheric disturbances (Vlasov et al., 2011). Figure 3c shows electron density computed from the ion line for the same period as 

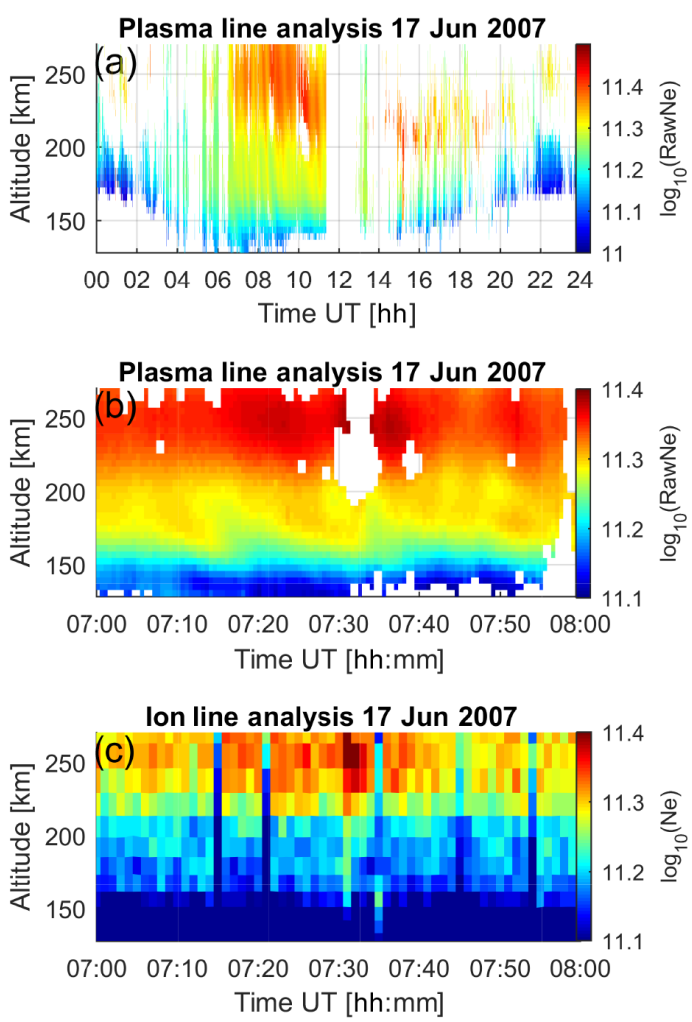

Figure 3. Electron density on 17 June 2007. Panels (a) and (b) show electron density recovered from $30 \mathrm{~s}$ integrated plasma line data. Panel (c) shows electron density resulting from ion line analysis with $60 \mathrm{~s}$ integration, performed with the GUISDAP tool (Lehtinen and Huuskonen, 1996).

in panel (b). The electron densities agree roughly with the ones in panel (b); however, it is evident that panel (b) shows greater detail at the cost of incomplete data coverage.

A detailed comparison of electron density recovered from the plasma line and the result from standard ion line analysis is shown in Fig. 4. Panel (a) shows the electron density at $195 \mathrm{~km}$ altitude on 17 June 2007, derived from plasma line measurements. In panel (b) the electron density profile is shown for 09:09:54 UT. The electron density profile is covered almost completely by the plasma line measurements (black points with error bars), corrected for the electron temperature obtained from the ion line analysis. The steps in electron density are due to the limited accuracy of and low resolution in electron temperature. Also shown is the result from the ion line analysis (pink asterisks). The discrepancy of the electron densities at $150-200 \mathrm{~km}$ altitude is likely due to the known problem of ion line fitting at these altitudes, where there is a transition of ion composition from molecular to atomic oxygen (Blelly et al., 2010).
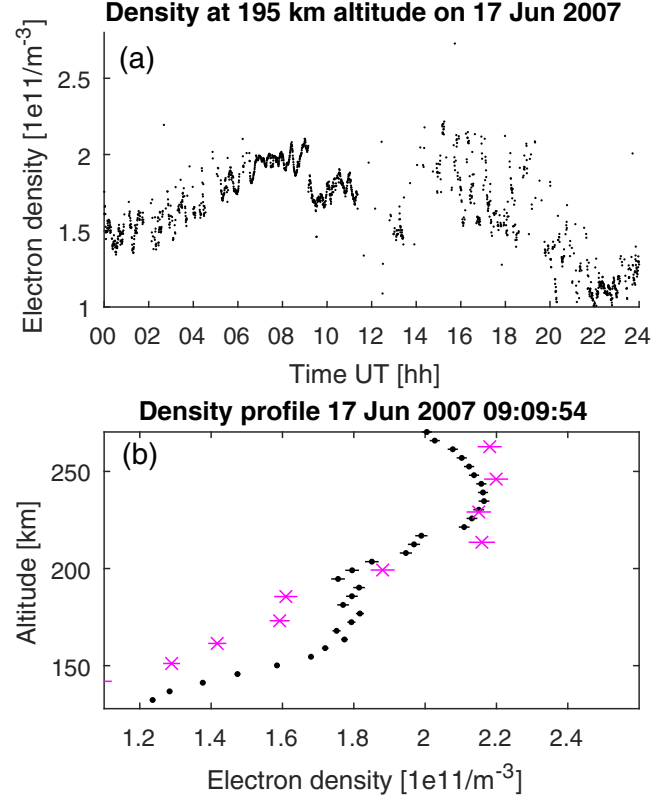

Figure 4. (a) Plasma density recovered from plasma line data using $30 \mathrm{~s}$ integration (black points). (b) Electron density profile from plasma line data (black) and from $60 \mathrm{~s}$ integrated ion line data (pink asterisks)

\section{Discussion}

The outlined method describes a non-iterative algorithm for recovering the range-frequency dependence of the plasma line backscatter. Since the algorithm is non-iterative a relatively short runtime is achieved and large datasets can be readily processed even on modern personal computers in short time. The algorithm processes 1 day of plasma line data acquired with the IPY experiment in less than $15 \mathrm{~min}$ and consequently 1 year of data in about 3.5 days.

For independent validation, the algorithm was run on a specific day (21 July 2017, $2 \mathrm{~h}$ between 04:00 and 06:00 UT, 1200 dumps of $6 \mathrm{~s}$ integration in total). Over $87 \%$ of profiles (308 dumps) detectable by a human were detected and correctly processed by the algorithm. The remaining $13 \%$ were partially detected or resulted in non-contiguous mask regions.

The example used to demonstrate the method outlines the capabilities of the algorithm, but also some weaknesses. The strongest features of the range-frequency dependence of the plasma line are successfully recovered. However, some weaker features, easily recognized by the human eye, are not detected. These non-recovered features are close to the noise level and can only be recognized due to their clustering. A cluster of points could, for example, be identified in the mask resulting from step F1, but since the detection of clustering would result in significantly increased computation time, this was not implemented. 


\section{Conclusions}

Plasma lines are only detectable when enhanced over thermal levels by photoelectrons produced by solar EUV irradiation, by secondary superthermal electrons caused by electron precipitation, or by other processes. The data produced by alternating code experiments often exhibit clutter due to the variability of the targets. We have presented a detailed method to declutter such alternating code data in order to produce a clean measurement of the plasma line frequency shift. The method has been used for ESR data from the IPY year 20072008. Plasma line backscatter was detected in $22.6 \%$ of the data, with higher detectability in the summer season (peaking at $90 \%$ for daytime $\mathrm{F}$ region).

The advantage of plasma line measurements compared with ion line data is demonstrated with an event where ionospheric disturbances related to atmospheric gravity waves are resolved, thanks to the higher time and range resolution. The drawback of the plasma line technique to measure electron density in the ionosphere is the incomplete data coverage; however, whenever plasma line data are available the method is superior, and therefore plasma line analysis should be conducted in addition to ion line analysis when possible. A comparison of large statistical sets of plasma density observations (see Sojka et al., 2009; He et al., 2012) is a natural application of this study, but this is outside the scope of this report. We have not focused here on strongly enhanced plasma lines, related to strong Langmuir turbulence (e.g. Schlatter et al., 2014), which are a topic of separate study.

Data availability. Data are available through EISCAT (https:// www.eiscat.se/scientist/data/).

Competing interests. The authors declare that they have no conflict of interest.

Acknowledgements. EISCAT is an international association supported by research organizations in China (CRIRP), Finland (SA), Japan (NIPR and STEL), Norway (NFR), Sweden (VR), and the United Kingdom (NERC). The continuous ESR run during the IPY was supported by Norway (NFR) and the USA (NSF). Hanna Dahlgren is supported by the Swedish Research Council under grant 350-2012-6591.

The topical editor, Steve Milan, thanks Asti Bhatt and two anonymous referees for help in evaluating this paper.

\section{References}

Akbari, H., Bhatt, A., La Hoz, C., and Semeter, J. L.: Incoherent Scatter Plasma Lines: Observations and Applications, Space Sci. Rev., https://doi.org/10.1007/s11214-017-0355-7, online first, 2017.
Bauer, P., Lejeune, G., and Cole, K. D.: Field-aligned electric currents and their measurement by the incoherent backscatter technique, Planet. Space Sci., 24, 479-485, https://doi.org/10.1016/0032-0633(76)90093-3, 1976.

Behnke, R. A. and Ganguly, S.: First direct groundbased measurements of electron drift in the ionospheric F region, J. Geophys. Res., 91, 10178-10182, https://doi.org/10.1029/JA091iA09p10178, 1986.

Blelly, P.-L., Alcaydé, D., and van Eyken, A. P.: A new analysis method for determining polar ionosphere and upper atmosphere characteristics from ESR data: Illustration with IPY period, J. Geophys. Res.-Space, 115, A09322, https://doi.org/10.1029/2009JA014876, 2010.

Djuth, F. T., Sulzer, M. P., and Elder, J. H.: Application of the coded long-pulse technique to plasma line studies of the ionosphere, Geophys. Res. Lett., 21, 2725-2728, https://doi.org/10.1029/94GL01699, 1994.

Djuth, F. T., Sulzer, M. P., Elder, J. H., and Wickwar, V. B.: High-resolution studies of atmosphere-ionosphere coupling at Arecibo Observatory, Puerto Rico, Radio Sci., 32, 2321-2344, https://doi.org/10.1029/97RS02797, 1997.

Dougherty, J. P. and Farley, D. T.: A Theory of Incoherent Scattering of Radio Waves by a Plasma, P. Roy. Soc. Lond. A Mat., 259, 79-99, https://doi.org/10.1098/rspa.1960.0212, 1960.

Guio, P. and Lilensten, J.: Effect of suprathermal electrons on the intensity and Doppler frequency of electron plasma lines, Ann. Geophys., 17, 903-912, https://doi.org/10.1007/s00585999-0903-x, 1999.

Hagfors, T. and Lehtinen, M.: Electron temperature derived from incoherent scatter radar observations of the plasma line frequency, J. Geophys. Res.-Space, 86, 119-124, https://doi.org/10.1029/JA086iA01p00119, 1981.

He, F., Zhang, B., and Huang, D.: Averaged NmF2 of cusplatitude ionosphere in northern hemisphere for solar minimum - Comparison between modeling and ESR during IPY, Science China Technological Sciences, 55, 1281-1286, https://doi.org/10.1007/s11431-012-4782-0, 2012.

Kirkwood, S. and Bjorna, N.: Electron temperatures determined by tristatic plasma-line observations with the EISCAT UHF incoherent scatter radar, Geophys. Res. Lett., 19, 661-664, https://doi.org/10.1029/92GL00523, 1992.

Lehtinen, M. S. and Häggström, I.: A new modulation principle for incoherent scatter measurements, Radio Sci., 22, 625-634, https://doi.org/10.1029/RS022i004p00625, 1987.

Lehtinen, M. S. and Huuskonen, A.: General incoherent scatter analysis and GUISDAP, J. Atmos. Terr. Phys., 58, 435-452, https://doi.org/10.1016/0021-9169(95)00047-X, 1996.

Nicolls, M. J., Sulzer, M. P., Aponte, N., Seal, R., Nikoukar, R., and González, S. A.: High-resolution electron temperature measurements using the plasma line asymmetry, Geophys. Res. Lett., 33, 118107, https://doi.org/10.1029/2006GL027222, 2006.

Nilsson, H., Kirkwood, S., Lilensten, J., and Galand, M.: Enhanced incoherent scatter plasma lines, Ann. Geophys., 14, 1462-1472, https://doi.org/10.1007/s00585-996-1462-z, 1996.

Schlatter, N. M., Ivchenko, N., and Häggström, I.: On the relation of Langmuir turbulence radar signatures to auroral conditions, J. Geophys. Res.-Space, 119, 8499-8511, https://doi.org/10.1002/2013JA019457, 2014. 
Sojka, J. J., Nicolls, M. J., Heinselman, C. J., and Kelly, J. D.: The PFISR IPY observations of ionospheric climate and weather, J. Atmos. Sol.-Terr. Phy., 71, 771-785, https://doi.org/10.1016/j.jastp.2009.01.001, 2009.

Van Trees, H. L.: Detection, estimation, and modulation theory, John Wiley \& Sons, New York, USA, 2004.

Vierinen, J., Bhatt, A., Hirsch, M. A., Strømme, A., Semeter, J. L., Zhang, S.-R., and Erickson, P. J.: High temporal resolution observations of auroral electron density using superthermal electron enhancement of Langmuir waves, Geophys. Res. Lett., 43, 5979-5987, https://doi.org/10.1002/2016GL069283, 2016.
Vlasov, A., Kauristie, K., van de Kamp, M., Luntama, J.-P., and Pogoreltsev, A.: A study of Traveling Ionospheric Disturbances and Atmospheric Gravity Waves using EISCAT Svalbard Radar IPY-data, Ann. Geophys., 29, 2101-2116, https://doi.org/10.5194/angeo-29-2101-2011, 2011. 